This paper was published in the Journal of the Optical Society of America A and is made available as an electronic reprint with the permission of OSA. The paper can be found at the following URL on the OSA website:

http://www.opticsinfobase.org/josaa/abstract.cfm?uri=josaa-29-12-2667 or

http://www.opticsinfobase.org/josaa/viewmedia.cfm?uri=josaa-29-12-2667\&seq=0 . Systematic or multiple reproduction or distribution to multiple locations via electronic or other means is prohibited and is subject to penalties under law

Please cite this article as follows:

Boone, M. N., Devulder, W., Dierick, M., Brabant, L., Pauwels, E. and Van Hoorebeke, L. (2012). "Comparison of two single-image phase-retrieval algorithms for in-line x-ray phasecontrast imaging." Journal of the Optical Society of America A 29(12): 2667-2672. 


\title{
Comparison of two single-image phase-retrieval algorithms for in-line x-ray phase-contrast imaging
}

\author{
Matthieu N. Boone, ${ }^{1, *}$ Wouter Devulder, ${ }^{2}$ Manuel Dierick, ${ }^{1}$ Loes Brabant, ${ }^{1}$ \\ Elin Pauwels, ${ }^{1}$ and Luc Van Hoorebeke ${ }^{1}$ \\ ${ }^{1}$ Ghent University, Department of Physics and Astronomy Proeftuinstraat 86, Gent B-9000, Belgium \\ ${ }^{2}$ Ghent University, Department of Solid State Sciences Krijgslaan 281 S1, Gent B-9000, Belgium \\ *Corresponding author: Matthieu.Boone@UGent.be
}

Received August 3, 2012; revised October 26, 2012; accepted October 28, 2012;

posted October 31, 2012 (Doc. ID 173766); published November 28, 2012

\begin{abstract}
The attenuation of x-rays in a material forms the basis of x-ray radiography and tomography. By measuring the transmission of the $\mathrm{x}$-rays over a large amount of raypaths, the three-dimensional (3D) distribution of the x-ray linear attenuation coefficient can be reconstructed in a 3D volume. In x-ray microtomography ( $\mu \mathrm{CT})$, however, the $\mathrm{x}$-ray refraction yields a significant signal in the transmission image and the $3 \mathrm{D}$ distribution of the refractive index can be reconstructed in a 3D volume. To do so, several methods exist, on both a hardware and software level. In this paper, we compare two similar software methods, the modified Bronnikov algorithm and the simultaneous phase-and-amplitude retrieval. The first method assumes a pure phase object, whereas the latter assumes a homogeneous object. Although these assumptions seem very restrictive, both methods have proven to yield good results on experimental data. (C) 2012 Optical Society of America
\end{abstract}

OCIS codes: $\quad 340.7440,070.0070,100.3190$

\section{INTRODUCTION}

For more than 100 years, the attenuation of x-rays has been used in x-ray radiography and tomography to provide image contrast. During the past two decades, the refraction of x-rays by the object under investigation has gained interest due to its high sensitivity. At first, the effect was investigated at synchrotron facilities due to the high temporal coherence of their x-ray beam, but it quickly became evident at x-ray tubes with high spatial coherence [1-3]. Through the years, several methods to retrieve the phase information were developed using, e.g., grating interferometers [4-6], images at different propagation distances [7,8], and single-image phase-propagation imaging (PPI) [9-11]. In this paper, we will focus on the latter method, using no specific hardware tools for phase-contrast imaging.

In single-image PPI, the angular deviation of the refracted beam is exploited for contrast generation. This gives rise to a black-white fringe at the interface between two phases [3]. Although this contrast as such can be useful for imaging [12-15], it leads to unreal features on the reconstructed volume that are often unwanted. A widely used theoretical starting point to describe the phase-contrast effect is the transport of intensity equation (TIE) [16]:

$$
\frac{2 \pi}{\lambda} \frac{\partial I_{\theta}(\boldsymbol{r}, \boldsymbol{z})}{\partial z}=-\nabla_{\perp}\left[I_{\theta}(\boldsymbol{r}, \boldsymbol{z}) \nabla_{\perp} \phi_{\theta}(\boldsymbol{r}, \lambda)\right]
$$

where $I_{\theta}(\boldsymbol{r}, \boldsymbol{z})$ is the intensity measured after a propagation distance $z, \lambda$ is the x-ray wavelength, and $\phi_{\theta}$ is the projected phase shift at rotation step $\theta$. This projected phase shift can be expressed as

$$
\phi_{\theta}(\boldsymbol{r}, \lambda)=\frac{2 \pi}{\lambda} \int_{\text {raypath }} \delta\left(x^{\prime}, y^{\prime}, z^{\prime}, \lambda\right) \partial x^{\prime} \partial y^{\prime} \partial z^{\prime}
$$

where the integral is taken over the raypath from the x-ray source to the detector element, and $\delta(\lambda)$ is the decrement of the real part of the wavelength-dependent refractive index $n(\lambda)$ :

$$
n(\lambda)=1-\delta(\lambda)+i \beta(\delta) .
$$

The imaginary part of $n(\lambda)$ is the attenuation index $\beta$, which is related to the linear attenuation coefficient $\mu$ :

$$
\mu(x, y, z, \lambda)=\frac{2 \pi}{\lambda} \beta(x, y, z, \lambda) .
$$

Similar to the phase shift (2), a projected attenuation coefficient at rotation $\theta$ can be defined:

$$
\mu_{\theta}(\boldsymbol{r}, \lambda)=\int_{\text {raypath }} \mu\left(x^{\prime}, y^{\prime}, z^{\prime}, \lambda\right) \partial x^{\prime} \partial y^{\prime} \partial z^{\prime} .
$$

Equation (1) can be written as [17]

$$
\begin{aligned}
& I_{\theta}(z=d) \approx I_{\theta}(z=0) \\
& \quad \times\left[1-\frac{\lambda z}{2 \pi M} \nabla^{2} \phi_{\theta}+\frac{\lambda z}{2 \pi M}\left(\frac{\partial \mu_{\theta}}{\partial x} \frac{\partial \phi_{\theta}}{\partial x}+\frac{\partial \mu_{\theta}}{\partial y} \frac{\partial \phi_{\theta}}{\partial y}\right)\right],
\end{aligned}
$$

where the geometrical magnification $M$ has been added [11].

Under the assumption that the projected attenuation coefficient $\mu_{\theta}$ only varies slowly, Eq. (6) simplifies to $[\underline{7}, \underline{17}]$

$$
I_{\theta}(z=d) \approx I_{\theta}(z=0)\left(1-\frac{\lambda z}{2 \pi M} \nabla^{2} \phi_{\theta}\right) \text {. }
$$

It can be shown that, starting from Eq. (7), a filtered backprojection (FBP) algorithm can be applied to retrieve the spatial distribution of $\delta(x, y, z)$, using the filter $Q(\xi, \eta)[18,19]$ : 


$$
Q(\xi, \eta)=\frac{|\xi|}{\xi^{2}+\eta^{2}+\alpha}
$$

where $\xi$ and $\eta$ are the spatial frequency variables along $x$ and $y$, respectively. The free parameter $\alpha$ compensates for the remaining attenuation [19]. The filter (8) is applied on the Fourier transform of the adapted projection data $g_{\theta}(\boldsymbol{r})$

$$
g_{\theta}(\boldsymbol{r})=\frac{I_{\theta}(\boldsymbol{r}, \boldsymbol{z})}{I_{\theta}(\boldsymbol{r}, z=0)}-1,
$$

where $I_{\theta}(\boldsymbol{r}, z=0)$ is the pure attenuation image, which can be approximated by 1 for low-attenuating samples. In Eq. ( $\underline{8}), \alpha$ is a free parameter to compensate for the remaining attenuation [19]. In the following, this algorithm will be called the modified Bronnikov algorithm (MBA).

A different approach for phase retrieval can be found in [20], which assumes a homogeneous object. This assumption simplifies both Eqs. (2) and (5) to a projected thickness $T_{\theta}(\boldsymbol{r})$ multiplied by $\delta$ and $\mu$, respectively. It can be shown [20,21] that this projected thickness $T_{\theta}(\boldsymbol{r})$ can be retrieved from a single image:

$$
T_{\theta}(\boldsymbol{r})=-\frac{1}{\mu} \ln \left[\mathcal{F}^{-1}\left(\mu \frac{\mathcal{F}\left\{M^{2} I_{\theta}(M \boldsymbol{r}, z)\right\}}{z \delta\left(\xi^{2}+\eta^{2}\right) / M+\mu}\right)\right],
$$

which will be called the simultaneous phase-and-amplitude retrieval (SPAR) in the following. Although MBA and SPAR are very similar, the main practical difference lies in the image that is filtered. The Fourier filter of the SPAR algorithm is applied directly on normalized projection data, whereas Eq. (9) needs to be applied first in the MBA algorithm. In practice, this cannot be calculated exactly, but it is approximated by the normalized image decremented by 1 . There is no physical relevance for the remaining attenuation in this decremented image, causing artifacts in the reconstruction.
Next to these methods, several other algorithms for singleimage in-line phase retrieval exist [22-24]. Of these methods, MBA and SPAR are the most widely applicable [25] and are used on a regular basis at the Ghent University Center for Tomography (UGCT) [26] and at other facilities [27,28] for various applications [29,30]. Both methods are implemented in the in-house developed commercially available software package Octopus (inCT, Belgium, [31]). Despite the assumptions made in the derivations, a pure phase object for MBA or a homogeneous object for SPAR, both methods yield good results in practice, removing the phase artifacts and increasing the signal-to-noise ratio [32,33]. However, both methods have been reported to introduce significant blurring in the filtered images [26,27]. In this paper, a quantitative comparison of both methods is made to check the validity of these methods by comparing reconstructed slices of simulated data to the original slice and by evaluating the modulation transfer function (MTF) of the edge of these slices.

\section{MATERIALS AND METHODS}

In this paper, both simulated and experimental data containing mixed phase-and-amplitude projection images are reconstructed using MBA and SPAR. Simulated data are generated using an analytical projection simulator [34], calculating integrated raypath profiles (2) and (5) of an object consisting of a number of spherical objects [Fig. 1(a)]. The phase contrast was calculated using the second-order approximation ( $\underline{6})$ of the TIE (1). This way, several datasets of an object consisting of several nonconcentric spheres with attenuation coefficients $\mu_{1}$ and $\mu_{2}$, and refractive index decrement $\delta_{1}$ and $\delta_{2}$, were simulated for various attenuation levels between $1 \%$ and $90 \%$ :

1. $\mu_{2}=\mu_{1}, \delta_{2}=\delta_{1}$; from now on called a homogeneous phantom.

2. $\mu_{2}=2 \cdot \mu_{1}, \delta_{2}=2 \cdot \delta_{1}$; a semihomogeneous phantom.

3. $\mu_{2}=1.5 \cdot \mu_{1}, \delta_{2}=\delta_{1}$; a phantom with an attenuation inclusion
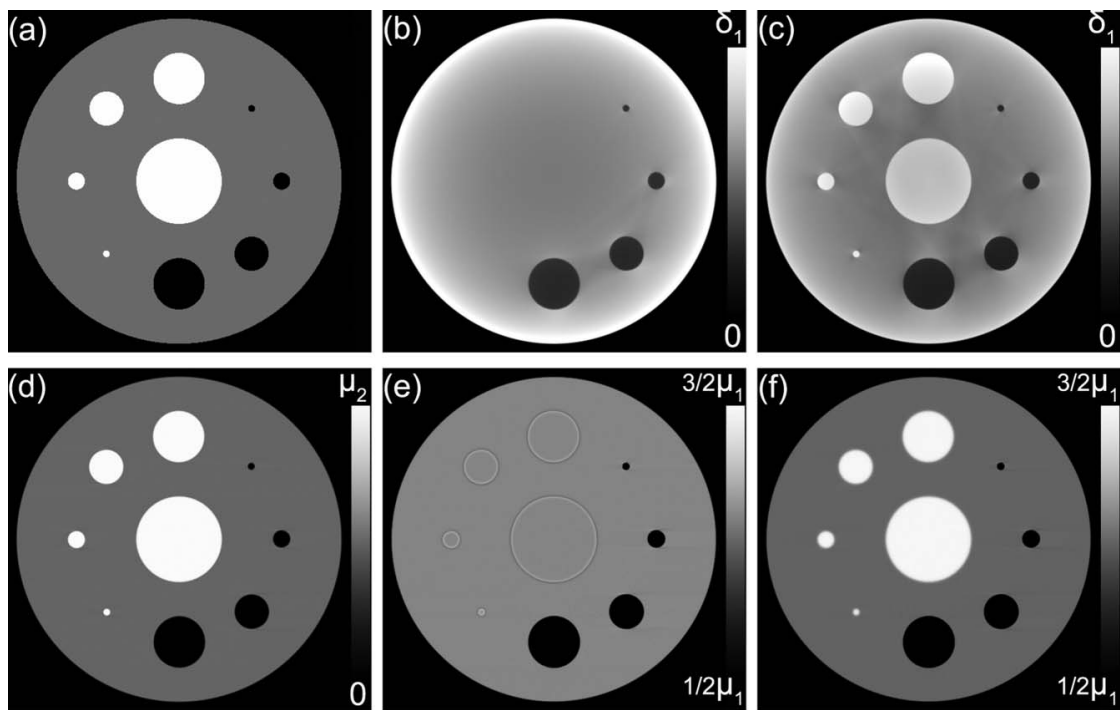

Fig. 1. (a) Original phantom, where black indicates air, gray indicates the matrix material with parameters $\mu_{1}$ and $\delta_{1}$, and white indicates the inclusions with parameters $\mu_{2}$ and $\delta_{2}$. (b) MBA reconstruction of the homogeneous object at 75\% attenuation. (c) MBA reconstruction of the semihomogeneous object at $75 \%$ attenuation. (d) SPAR reconstruction of the semihomogeneous object at $75 \%$ attenuation. (e) SPAR reconstruction of the object with phase inclusions at $25 \%$ attenuation. (f) SPAR reconstruction of the object with attenuation inclusions at $25 \%$ attenuation, reconstructed using SPAR 
4. $\mu_{2}=\mu_{1}, \delta_{2}=1.5 \cdot \delta_{1}$; a phantom with a phase inclusion.

The simulated projection image size is $512 \times 512$ pixels $^{2}$, which is also the size of the reconstruction grid. The reconstructed slices are compared to the original ones and the normalized root-mean-square error (NRMSE) is calculated. Additionally, edge profiles are calculated in polar coordinates to evaluate sharpness and remaining phase effects.

Experimental data was recorded at the modular micro-CT setup [35] of the (UGCT, http://www.UGCT.UGent.be). The object was a homogeneous cylinder of epoxy resin with a diameter of approximately $5 \mathrm{~mm}$, containing some air bubbles. It is not perfectly cylindrical, and has a smooth surface. It was scanned using the FeinFocus FXE160.51 transmission type x-ray tube head, operated at different tube voltages and hardware filters to achieve various attenuation levels (Table 1). A hardware collimator is applied to minimize secondary radiation effects [36]. The sample is rotated on a Micos UPR160-F Air high-precision stage, and the images are acquired using a Varian PaxScan $2520 \mathrm{~V}$ flat-panel detector. The tomographic reconstruction is performed using Octopus.

Table 1. Experimental Settings for the Epoxy Cylinder Scans

\begin{tabular}{lccc}
\hline Tube Voltage & $50 \mathrm{kV}_{p}$ & $90 \mathrm{kV}_{p}$ & $120 \mathrm{kV}_{p}$ \\
\hline Hardware filter & - & - & $1 \mathrm{~mm} \mathrm{Al}$ \\
Minimal transmission & $76.9 \pm 0.5 \%$ & $82.2 \pm 0.4 \%$ & $87.2 \pm 0.3 \%$ \\
\hline
\end{tabular}

\section{RESULTS}

\section{A. Simulated Data}

The NRMSE of the reconstruction for the phantoms with constant ratio $\mu_{i} / \delta_{i}$ as a function of the maximal attenuation is shown in Fig. 2(a). Due to this constant ratio, the NRMSE can be calculated with either $\mu$ or $\delta$ for reference. It can be easily seen that MBA performs worse for increasing attenuation, resulting in a cupping artifact [Figs. 1(b) and 1(c)]. On the other hand, the FBP reconstruction performs better at increasing attenuation, because the relative contribution of the phase artifacts decreases. The exact crossing point is highly dependent on the magnitude of the phase effect. The NRMSE of the SPAR reconstructions remains constant over the complete attenuation range as illustrated in Fig. 1(d). As this is the case for both the homogeneous phantom and the semihomogeneous phantom, it is clear that the assumption of SPAR can be extended to a constant ratio $\mu_{i} / \delta_{i}$, as can also be deduced from Eq. (10). Compared to the homogeneous object, the NRMSE has increased for all three reconstruction algorithms. This is most probably due to the increased number of features present in the semihomogeneous object.

For the phantom with the phase inclusion, the results are shown in Fig. 2(d). All three algorithms show lower NRMSE when compared to the attenuation coefficient than when compared to the refractive-index decrement. This indicates that all three algorithms tend to reconstruct the attenuation coefficient, which can be observed in Figs. $\underline{1(\mathrm{e})}$ and $\underline{1(\mathrm{f})}$. A similar effect can be observed for the sample with the
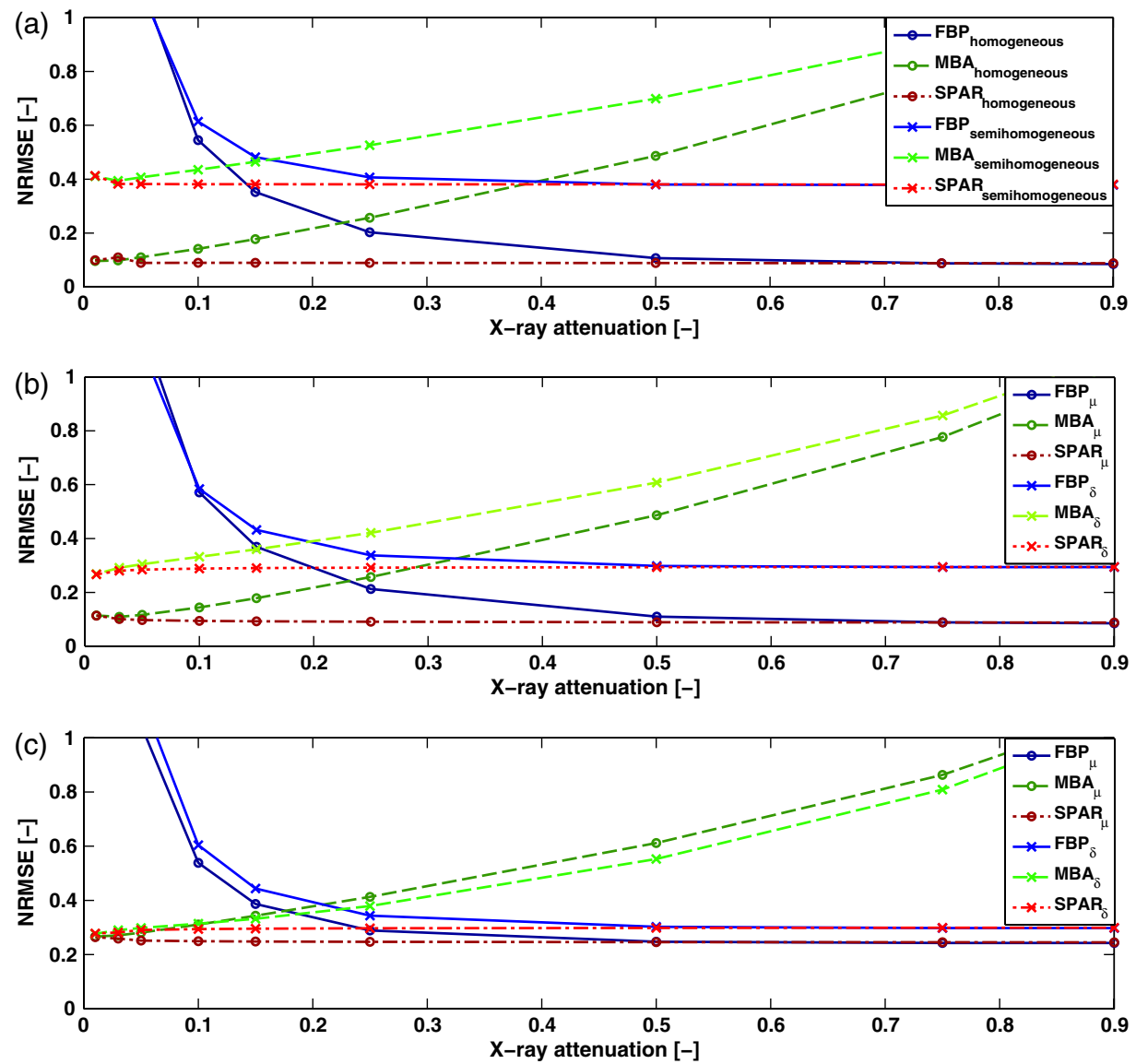

Fig. 2. (Color online) NRMSE for (a) homogeneous and semihomogeneous object. (b) Object with phase inclusion. (c) Object with attenuation inclusion. 
attenuation inclusion [Fig. 2(c)], except for the MBA reconstruction at high attenuations. This is caused by the cupping effect at the central inclusion.

To evaluate the reconstructed sharpness, the MTF is calculated in polar coordinates for the outer edge of the homogeneous sample. The $\mathrm{MTF}_{50 \%}$, where the MTF reaches $50 \%$ of its maximum value, is shown in Fig. 3 as a function of the maximal attenuation in the projection images. The three previously mentioned algorithms are compared to FBP on a simulation where no phase shift is added. It can be observed that the spatial resolution of the FBP reconstruction degrades as the attenuation decreases and thus contribution of the phase artifacts increases. Inversely, the resolution of the MBA reconstruction degrades as the attenuation increases. The resolution of the SPAR reconstructions remains constant over the investigated attenuation range.

\section{B. Experimental Data}

The line profile through the real object is given in Fig. 4(a) for three MBA reconstructions and in Fig. 4(b) for the corresponding SPAR reconstructions. The cupping effect in the MBA reconstructions can also be observed in this real object, whereas cupping is negligible in the SPAR reconstruction. The remaining cupping effect can be attributed to beam hardening, and is also present in the FBP reconstruction (not shown).

\section{DISCUSSION}

From these results, particularly from Figs. 2(b) and 2(c), it can be concluded that the condition of a homogeneous object or a semihomogeneous object should be met for SPAR, as expected from the derivation of the filter, but also for MBA. Both phase-retrieval algorithms show similar artifacts when this condition is not met. For objects with phase inclusions, the phase artifacts are not removed, but instead they are heavily blurred [Fig. 2(e)]. Surprisingly, MBA thus does not reconstruct the refractive-index decrement, but rather the attenuation coefficient. A similar behavior can be observed for the object with attenuation inclusions. Both MBA and SPAR reconstruct the attenuation coefficient rather than the refractive-index decrement, but the inclusions are blurred due to the inhomogeneity [Fig. 1(f)].

The free parameter $\alpha$ in the MBA algorithm affects greatly the reconstruction quality. Although methods exist to determine the optimal parameter value (e.g., [28]), it was chosen manually for these simulations such that the phase effect at the outer edge disappeared completely. Although this solution did not always yield the minimal NRMSE, the deviation from this minimal NRMSE never exceeded a few percent (data not shown).

The simulation formula (6) is an approximation of the physical phase-contrast effect and can as such be responsible

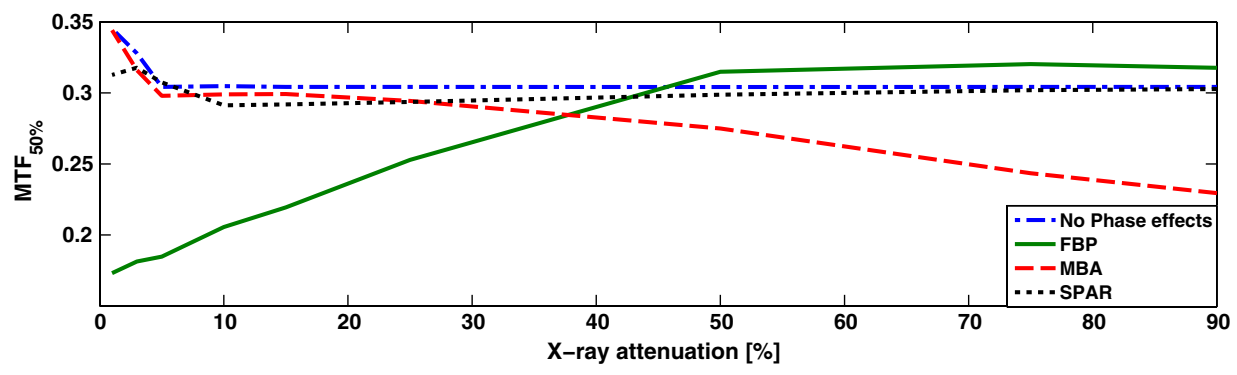

Fig. 3. (Color online) Calculated $\mathrm{MTF}_{50 \%}$ for the homogeneous sample at varying attenuation level.
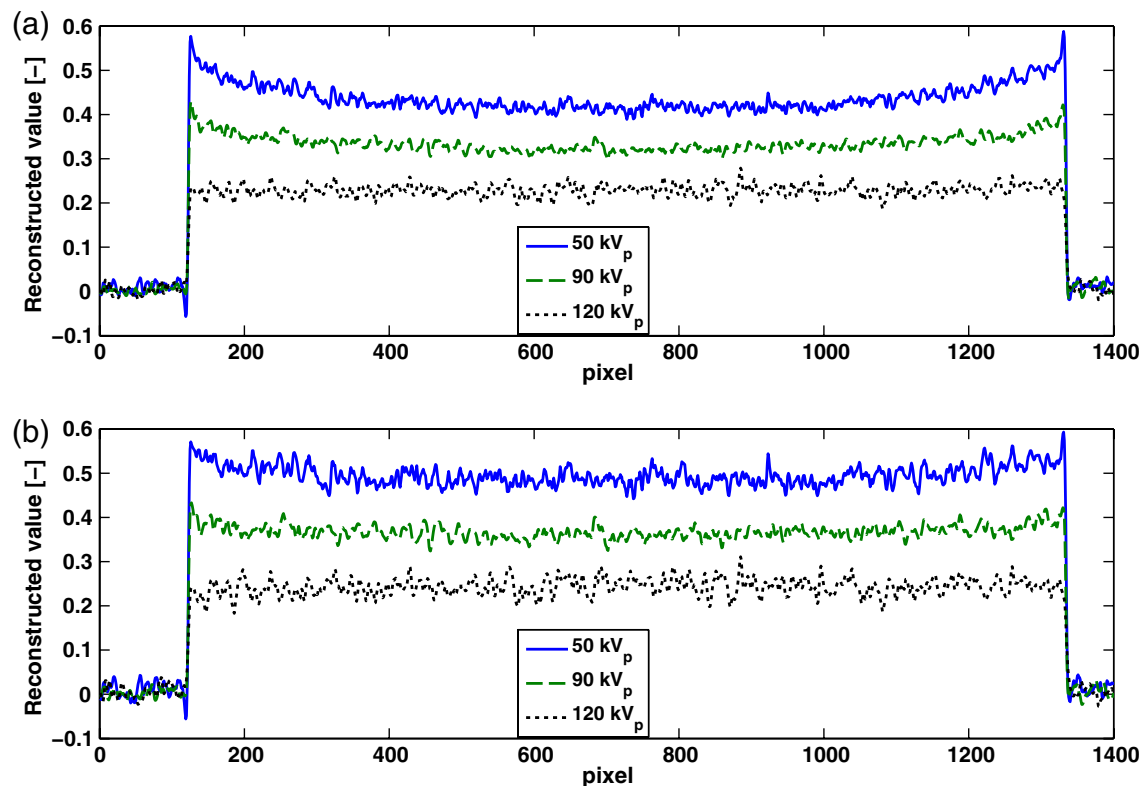

Fig. 4. (Color online) Line profile through the real homogeneous object scanned at $50 \mathrm{kV}_{p}, 90 \mathrm{kV}_{p}$, and $120 \mathrm{kV}_{p}$, respectively. (a) MBA reconstructions and (b) SPAR reconstructions. 
for the reconstruction artifacts. However, this formula is obtained through various derivations and approximations $[11,37,38]$. These approximations do not affect the behavior of the model with increasing attenuation of the model and are thus believed to have little influence on the conclusions drawn from Fig. 2. Furthermore, the artifacts observed in the simulated data are also observed in experimental data, strengthening this belief.

The simulated phantoms used in this paper are a simplification for real objects, representing limiting cases for real objects. The homogeneous phantom represents a single-material object. In such an object, different reconstructed values can occur due to changes in material density, which is represented by the semihomogeneous phantom. Pure attenuation or pure phase inclusions do not exist, but these results represent objects where $\mu_{1} / \delta_{1}<\mu_{2} / \delta_{2}$ and $\mu_{1} / \delta_{1}>\mu_{2} / \delta_{2}$, respectively. This is illustrated by Fig. 5, which shows a small Savonniéres limestone sample with water [39], imaged at the TOMCAT beamline of the synchrotron radiation facility Swiss Light Source $[\underline{40}, \underline{41}]$ and reconstructed using the SPAR algorithm. No additional filtering or segmentation steps are performed during reconstruction. The different phases of the rock matrix consist of the same material, but have different densities due to microporosity. The water inside the sample can be considered a phase inclusion since $\delta_{\text {water }} / \mu_{\text {water }}>\delta_{\text {rock }} / \mu_{\text {rock }}[\underline{42}$ ]; hence the phase artifacts are not completely removed. One solution to cope with such heterogeneous objects is proposed by $[43,44]$, where a filtering is performed for each different interface. To combine these different datasets, however, a segmentation of the different phases has to be made.

In Fig. 3, the $\mathrm{MTF}_{50 \%}$ of the MBA reconstruction degrades as attenuation increases. However, this is of minor importance as the cupping effect has already deteriorated the image quality at this point. Despite previous reports of a significant blurring of both MBA and SPAR even for low-attenuating objects, these measurements are not conclusive to confirm this. Three reasons contribute to this inconsistency. First, previous reports were based on visual observations. Since phase-contrast effects can be seen as an edge-enhancement effect, the FBP reconstructions tend to be evaluated sharper than other reconstructions. Second, these results are based on simulated data, obtained by an approximation of the TIE. Other effects can contribute to real data, degrading the results of the phase-retrieval algorithms. Third, these

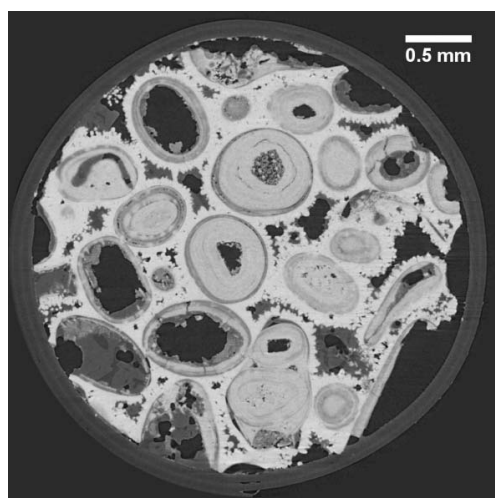

Fig. 5. Reconstruction using SPAR of a rock sample showing the remaining phase effects due to sample inhomogeneity. results are obtained from a homogeneous object. As mentioned earlier, the inclusions in the heterogeneous objects show blurring. This indicates that heterogeneity or mismatch in filtering parameters also leads to blurring.

The results in Fig. $\underline{4}$ are in good correspondence with the simulated data. However, altering the attenuation of the sample by adjusting the mean detected photon energy can introduce other effects as well, such as an alteration of $\mu / \delta$, and thus require different retrieval parameters for SPAR.

\section{CONCLUSION}

Both single-image in-line phase-retrieval algorithms perform very similarly on low-attenuating samples. However, as attenuation increases, MBA performs worse, resulting in a cupping effect. This effect is due to a violation of the assumption of a pure phase object. The quality of the SPAR reconstructions, on the other hand, remains constant. Both algorithms fail at reconstructing heterogeneous objects, where phase artifacts are left uncorrected or attenuating inclusions are blurred. Both SPAR and MBA reconstruct the attenuation coefficient, or a value proportional to this coefficient. This is particularly surprising for MBA, because it is derived as a phase-retrieval algorithm. Considering the similar workflow, it can be concluded that SPAR is to be preferred over MBA, based on the performance for highly attenuating samples.

\section{ACKNOWLEDGMENTS}

The authors gratefully acknowledge Hannelore Derluyn (EMPA) for providing the limestone sample and Peter Moddregger for the assistance at the TOMCAT beamline. The BOF-UGent is acknowledged for the doctoral grant to Loes Brabant and the financial support (GOA 01G01008).

\section{REFERENCES}

1. T. J. Davis, D. Gao, T. E. Gureyev, A. W. Stevenson, and S. W. Wilkins, "Phase-contrast imaging of weakly absorbing materials using hard x-rays," Nature (London) 373, 595-598 (1995).

2. P. Cloetens, R. Barrett, J. Baruchel, J. P. Guigay, and M. Schlenker, "Phase objects in synchrotron radiation hard x-ray imaging," J. Phys. D: Appl. Phys. 29, 133-146 (1996).

3. S. W. Wilkins, T. E. Gureyev, D. Gao, A. Pogany, and A. W. Stevenson, "Phase-contrast imaging using polychromatic hard x-rays," Nature 384, 335-338 (1996).

4. F. Pfeiffer, T. Weitkamp, O. Bunk, and C. David, "Phase retrieval and differential phase-contrast imaging with low-brilliance x-ray sources," Nat. Phys. 2, 258-261 (2006).

5. C. David, T. Weitkamp, F. Pfeiffer, A. Diaz, J. Bruder, T. Rohbeck, A. Groso, O. Bunk, M. Stampanoni, and P. Cloetens, "Hard x-ray phase imaging and tomography using a grating interferometer," Spectrochim. Acta: Part B 62, 626-630 (2007).

6. S. A. McDonald, F. Marone, C. Hintermuller, G. Mikuljan, C. David, F. Pfeiffer, and M. Stampanoni, "Advanced phasecontrast imaging using a grating interferometer,” J. Synchrotron Radiat. 16, 562-572 (2009)

7. P. Cloetens, W. Ludwig, J. Baruchel, D. Van Dyck, J. Van Landuyts, J. P. Guigay, and M. Schlenker, "Holotomography: quantitative phase tomography with micrometerresolution using hard synchrotron radiation x rays," Appl. Phys. Lett. 75, 2912-2914 (1999).

8. M. Langer, P. Cloetens, J. P. Guigay, and F. Peyrin, "Quantitative comparison of direct phase retrieval algorithms in in-line phase tomography," Med. Phys. 35, 4556-4566 (2008).

9. P. Cloetens, M. Pateyron-Salom, J. Y. Buffire, G. Peix, J. Baruchel, F. Peyrin, and M. Schlenker, "Observation of microstructure and damage in materials by phase sensitive radiography and tomography," J. Appl. Phys. 81, 5878-5886 (1997). 
10. A. Pogany, D. Gao, and S. W. Wilkins, "Contrast and resolution in imaging with a microfocus x-ray source,” Rev. Sci. Instrum. 68, 2774-2782 (1997).

11. A. Peterzol, A. Olivo, L. Rigon, S. Pani, and D. Dreossi, "The effects of the imaging system on the validity limits of the ray-optical approach to phase contrast imaging," Med. Phys. 32, 3617-3627 (2005).

12. C. M. Laperle, T. J. Hamilton, P. Wintermeyer, E. J. Walker, D. Shi, M. A. Anastasio, Z. Derdak, J. R. Wands, G. Diebold, and C. Rose-Petruck, "Low density contrast agents for x-ray phase contrast imaging: the use of ambient air for x-ray angiography of excised murine liver tissue," Phys. Med. Biol. 53, 6911-6923 (2008).

13. E. M. Friis, P. R. Crane, K. R. Pedersen, S. Bengton, P. C. J. Donoghue, G. W. Grimm, and M. Stampanoni, "Phasecontrast X-ray microtomography links cretaceous seeds with gnetales and bennettitales," Nature 450, 549-552 (2007).

14. P. Trtik, J. Dual, D. Keunecke, D. Mannes, P. Niemz, P. Stähli, A. Kaestner, A. Groso, and M. Stampanoni, "3d imaging of microstructure of spruce wood," J. Struct. Biol. 159, 46-55 (2007).

15. P. Tafforeau and T. M. Smith, "Nondestructive imaging of hominoid dental microstructure using phase contrast x-ray synchrotron microtomography," J. Hum. Evol. 54, 272-278 (2008).

16. M. R. Teague, "Deterministic phase retrieval: a Green's function solution,” J. Opt. Soc. Am. 73, 1434-1441 (1983).

17. A. V. Bronnikov, "Theory of quantitative phase-contrast computed tomography,” J. Opt. Soc. Am. A 19, 472-480 (2002).

18. A. V. Bronnikov, "Phase-contrast CT: fundamental theorem and fast image reconstruction algorithms," Proc. SPIE 6318, 63180Q (2006).

19. A. Groso, R. Abela, and M. Stampanoni, "Implementation of a fast method for high resolution phase contrast tomography," Opt. Express 14, 8103-8110 (2006).

20. D. M. Paganin, S. C. Mayo, T. E. Gureyev, P. R. Miller, and S. W. Wilkins, "Simultaneous phase and amplitude extraction from a single defocused image of a homogeneous object," J. Microsc. 206, 33-40 (2002).

21. S. C. Mayo, T. J. Davis, T. E. Gureyev, P. R. Miller, D. M. Paganin, A. Pogany, A. W. Stevenson, and S. W. Wilkins, "X-ray phasecontrast microscopy and microtomography," Opt. Express 11, 2289-2302 (2003).

22. T. E. Gureyev, T. J. Davis, A. Pogany, S. C. Mayo, and S. W. Wilkins, "Optical phase retrieval by use of first Born-andRytov-type approximations," Appl. Opt. 43, 2418-2430 (2004).

23. X. Wu, H. Liu, and A. Yan, "X-ray phase-attenuation duality and phase retrieval," Opt. Lett. 30, 379-381 (2005).

24. Y. De Witte, M. N. Boone, J. Vlassenbroeck, M. Dierick, and L. Van Hoorebeke, "Bronnikov-aided correction for x-ray computed tomography,” J. Opt. Soc. Am. A 26, 890-894 (2009).

25. A. Burvall, U. Lundstrm, P. A. C. Takman, D. H. Larsson, and H. M. Hertz, "Phase retreival in X-ray phase-contrast imaging suitable for tomography," Opt. Express 19, 10359-10376 (2011).

26. M. N. Boone, Y. De Witte, M. Dierick, J. Van den Bulcke, J. Vlassenbroeck, and L. Van Hoorebeke, "Practical use of the modified Bronnikov algorithm in micro-CT," Nucl. Instrum. Methods Phys. Res. Sect. B 267, 1182-1186 (2009).

27. T. Weitkamp, D. Haas, D. Wegrzynek, and A. Rack, "Ankaphase: software for single-distance phase retrieval from inline x-ray phase-contrast radiographs," J. Synchrotron. Radiat. 18, 617-629 (2011).

28. R. C. Chen, H. L. Xie, L. Rigon, R. Longo, E. Castelli, and T. Q Xiao, "Phase retrieval in quantitative $\mathrm{x}$-ray microtomography with a single sample-to-detector distance," Opt. Lett. $\mathbf{3 6}$, 1719-1721 (2011).

29. A. Almeida, S. Possemiers, M. N. Boone, T. De Beer, T. Quinten, L. Van Hoorebeke, J.-P. Remon, and C. Vervaet, "Ethylene vinyl acetate as matrix for oral sustained release dosage forms produced via hot-melt extrusion," Eur. J. Pharm. Biopharm. 77 , 297-305 (2011)

30. D. Derome, M. Griffa, M. Koebel, and J. Carmeliet, "Hysteretic swelling of wood at cellular scale probed by phase-contrast x-ray tomography,” J. Struct. Biol. 173, 180-190 (2011).

31. J. Vlassenbroeck, M. Dierick, B. Masschaele, V. Cnudde, L. Van Hoorebeke, and P. Jacobs, "Software tools for quantification of x-ray microtomography at the UGCT," Nucl. Instrum. Methods Phys. Res.: Sect. A 580, 442-445 (2007).

32. B. D. Arhatari, W. P. Gates, N. Estiaghi, and A. G. Peele, "Phase retrieval tomography in the presence of noise," J. Appl. Phys. 107, 034904 (2010).

33. M. N. Boone, Y. De Witte, M. Dierick, A. Almeida, and L. Van Hoorebeke, "Improved signal-to-noise ratio in laboratory-based phase contrast tomography," Microsc. Microanal. 18, 399-405 (2012).

34. Y. De Witte, "Improved and practically feasible reconstruction methods for high resolution x-ray tomography," Ph.D. thesis (Ghent University, 2010).

35. B. Masschaele, V. Cnudde, M. Dierick, P. Jacobs, L. Van Hoorebeke, and J. Vlassenbroeck, "UGCT: new x-ray radiography and tomography facility,” Nucl. Instrum. Methods Phys. Res. Sect. A 580, 266-269 (2007).

36. M. N. Boone, J. Vlassenbroeck, S. Peetermans, D. Van Loo, M Dierick, and L. Van Hoorebeke, "Secondary radiation in transmission-type $\mathrm{x}$-ray tubes: simulation, practical issues and solution in the context of x-ray microtomography," Nucl. Instrum. Methods Phys. Res. Sect. A 661, 7-12 (2012).

37. $\mathrm{X}$. $\mathrm{Wu}$ and $\mathrm{H}$. Liu, "A general theoretical formalism for X-ray phase contrast imaging," J. X-ray Sci. Technol. 11, 33-42 (2003).

38. T. E. Gureyev and S. W. Wilkins, "On x-ray phase imaging with a point source,” J. Opt. Soc. Am. A 15, 579-585 (1998).

39. H. Derluyn, M. Griffa, D. Mannes, I. Jerjen, J. Dewanckele, P. Vontobel, A. Sheppard, M. N. Boone, D. Derome, V. Cnudde, E. Lehmann, and J. Carmeliet, "Probing salt crystallization damage mechanisms in porous limestone with neutron radiography and x-ray tomography," in 5th International Building Physics Conference (2012), pp. 95-102.

40. M. Stampanoni, A. Groso, A. Isenegger, G. Mikuljan, Q. Chen, A Bertrand, S. Henein, R. Betemps, U. Frommherz, P. Bhler, D. Meister, M. Lange, and R. Abela, "Trends in synchrotron-based tomographic imaging: the SLS experience," Proc. SPIE 6318, 63180M (2006).

41. F. Marone, C. Hintermuller, S. A. McDonald, R. Abela, G. Mikuljan, A. Isenegger, and M. Stampanoni, "X-ray tomographic microscopy at TOMCAT,” J. Phys.: Conf. Ser. 186, 012042 (2009).

42. NIST, "X-ray form factor, attenuation and scattering tables," http://physics.nist.gov/PhysRefData/FFast/html/form.html.

43. M. A. Beltran, D. M. Paganin, K. Uesugi, and M. J. Kitchen, "2D and 3D x-ray phase retrieval of multi-material objects using a single defocus distance," Opt. Express 18, 6423-6436 (2010).

44. M. A. Beltran, D. M. Paganin, K. W. Siu, A. Fouras, S. B. Hooper D. H. Reser, and M. J. Kitchen, "Interface-specific x-ray phase retrieval tomography of complex biological organs," Phys. Med. Biol. 56, 7353-7369 (2011). 